\title{
Impact of glycemic control in diabetes mellitus on management of COVID-19 infection
}

\author{
Sudhir Bhandari ${ }^{1} \cdot$ Govind Rankawat $^{1}$ (I) $\cdot$ Ajeet Singh ${ }^{1} \cdot$ Vishal Gupta $^{1} \cdot$ Shivankan Kakkar $^{2}$
}

Received: 8 June 2020 / Accepted: 24 August 2020 / Published online: 2 September 2020

(C) Research Society for Study of Diabetes in India 2020

\begin{abstract}
Background Diabetes mellitus may be associated with increased severity and enhanced mortality in COVID-19 infections. The present study was undertaken to evaluate the clinical presentation, laboratory parameters, radiological imaging, management, and outcome of COVID-19 infection in patients of diabetes mellitus and its association with glycemic control.

Methods The present study was designed to evaluate the difference between uncontrolled and controlled diabetes for COVID-19 manifestations by enrolling 80 admitted COVID-19 patients. Patients were categorized into two groups, where group 1 had patients with uncontrolled diabetes as indicated by $\mathrm{HbAl} 1 \mathrm{c}>8 \mathrm{~g} \%$ and group 2 had patients with controlled diabetes as indicated by $\mathrm{HbA} 1 \mathrm{c}<$ $8 \mathrm{~g} \%$. Information concerning medical history, clinical manifestations, laboratory findings, radiological imaging, management, and outcome was extracted from medical records for evaluation, interpretation, and association among both the groups.

Results COVID-19 patients with uncontrolled diabetes exhibited a severe symptomatic presentation, excessive uncontrolled inflammatory responses, and hypercoagulable state. Total leukocyte count, neutrophil-lymphocyte ratio, serum levels of IL-6, FDP, and Ddimer were significantly raised $(p<0.05)$ in case of uncontrolled diabetes as compared with controlled diabetes. Radiological findings detected by chest radiograph and computed tomography chest suggested severe lung involvement in uncontrolled diabetes. COVID-19 patients with uncontrolled diabetes required intensive treatment as compared with controlled diabetes group in terms of insulin therapy $(p=0.0226)$ and non-invasive ventilation $(p=0.0292)$. Patients with uncontrolled diabetes had higher mortality $(p=0.0375)$ and required prolonged hospitalization $(p=0.0479)$ as compared with controlled diabetes group.

Conclusion From the current study, it can be concluded that uncontrolled diabetic condition might be a risk factor for severity and morbidity of COVID-19 patients. Uncontrolled diabetes mellitus might be responsible for an overall higher susceptibility for COVID-19 infection and severity in terms of symptomatic presentation, inflammatory storm, rapid pulmonary invasion, requirement of more intensive treatment, and a poor outcome.
\end{abstract}

Keywords COVID-19 · Diabetes mellitus $\cdot$ HbAlc $\cdot$ Glycemic control $\cdot$ Management

Govind Rankawat

govindrankawat@gmail.com

Sudhir Bhandari

drs_bhandari@yahoo.com

Ajeet Singh

dr.ajeetsingh@yahoo.com

Vishal Gupta

drvishalguptamd@rediffmail.com

Shivankan Kakkar

drshivankan@gmail.com

1 Department of General medicine, SMS Medical College and Attached Group of Hospital, Jaipur, Rajasthan, India

2 Department of Pharmacology, SMS Medical College and Attached Group of Hospital, Jaipur, Rajasthan, India

\section{Introduction}

Severe acute respiratory syndrome corona virus 2 (SARSCoV-2) was the pathogen behind coronavirus disease (COVID-19) that emerged from Wuhan in China and was rapidly spread across most of the nations worldwide. The disease presentation may range from an asymptomatic state to a severe pneumonia associated with acute lung failure [1]. COVID-19 incubation period may range from 2 to 14 days. The usual presentation in COVID-19-positive patients has been fever, cough, shortness of breath, fatigue, loss of appetite, sputum production, joint pain, nausea, vomiting, and diarrhea. A large number of patients may not exhibit noticeable symptoms. A severe disease could be associated with fatal complications such as pneumonia, acute respiratory distress 
syndrome (ARDS), multi-organ failure, septic shock, disseminated intravascular coagulation, and ultimately leading to death $[2,3]$. The total number of confirmed COVID-19 cases worldwide have risen to 6, 644, 011 with 391, 839 deaths as of 6 June 2020. India, a nation already been the diabetes capital of the world, has reported 236, 657 cases of COVID-19 with 6642 deaths [4].

Angiotensin-converting enzyme 2 (ACE2) has been identified as a surface receptor responsible for SARS coronavirus (SARS-Co-V) invasion in human cells with direct interaction with its spike glycoprotein (S protein) [5]. Moreover, a ten to twenty-fold higher affinity of ACE2 towards receptor-binding domain (RBD) of SARS-CoV-2 as compared with the RBD of SARS-Co-V has been suggested. This might be the plausible explanation of ACE2 serving as a receptor for SARS$\mathrm{CoV}-2$ invasion [6]. Diabetes mellitus has already been a leading cause of morbidity worldwide, that is capable of affecting almost each and every system of the body [7]. Consequently, a deregulated immune system might develop, predisposing to various infections in diabetic patients [8]. ACE2 has anti-inflammatory effects, and its expression is found reduced in patients of DM possibly due to glycosylation. This might explain the occurrence of a severe acute lung injury and ARDS in diabetic patients. This makes diabetic population with or without other comorbidities susceptible to a higher morbidity and mortality due to COVID-19. A severe disease in such patients requires intensive approach to manage COVID-19.

The prevalence of diabetes in India is high, and paucity of data on its association with COVID-19 warrants identification of factors responsible for severe outcome in such patients. The present study was designed in this context to evaluate the association between glycemic control in diabetes mellitus patients with progression and prognosis of COVID-19 in patients admitted to the S.M.S. Medical College and Attached Hospitals, Jaipur, a premier tertiary care center in India. The study was also aimed at evaluating the association of clinical presentation, severity of disease, management, and outcome in patients of diabetes mellitus with uncontrolled and controlled blood sugar levels.

\section{Method}

\section{Study design}

The present retrospective, observational study was conducted on eighty COVID-19 patients with known cases of type 2 diabetes mellitus (T2DM) already on antidiabetic medications, admitted to S.M.S. Medical College and Attached Hospitals, Jaipur, Rajasthan, India. Based upon the glycemic control, all patients of T2DM were categorized into two separate groups. Group 1 had T2DM patients with poorly controlled blood sugar levels as indicated by $\mathrm{HbA} 1 \mathrm{c}$ more than $8 \mathrm{~g} \%$. Group 2 included patients of T2DM, with controlled blood sugar levels as indicated by HbAlc less than $8 \mathrm{~g} \%$. Both of these groups were matched for age, gender, and absence of other comorbidities. In order to negate the implication of other comorbid conditions, we have taken into consideration COVID-19-positive patients with known to type 2 diabetes mellitus only and no other health-related chronic issues.

\section{Data collection}

The diagnosis of COVID-19 was based on the World Health Organization interim guidance, wherein confirmed cases were positive on reverse transcriptase polymerase chain reaction (RT-PCR) assay of nasal and pharyngeal swab specimens [1]. Patients of T2DM after exclusion of other comorbidities were segregated and categorized in two groups based on their glycemic control. Information regarding epidemiological data, medical history, clinical manifestations, laboratory findings, chest radiograph (CXR) findings, ultrasonography (USG) chest, high-resolution computed tomography (HRCT) scans of the chest, diabetic complications, treatment, and outcome was extracted from medical records of admitted patients. The laboratory findings were based upon hemogram, c-reactive protein (CRP), ferritin, fibrin degradation product (FDP), Ddimer, and interleukin-6 (IL-6). Radiological findings were inferred using average visual score from digital chest radiograph (CXR) (scored from 0 to 4 according to visual assessment of involved lung area) [9], average severity score from USG chest (classified on sliding scoring scale of severity on the basis of 14 zone severity scores ranging from 0 to 42) [10], CT severity score from HRCT chest (assigned out of 25 based upon percentage area involved in each of the 5 lobes) [11], and proportion of patients with CT severity scores $>10 / 25$. The data of patients with diabetes complications, particularly diabetic ketoacidosis and septic shock, was collected. Data regarding treatment among both groups by the use of hydroxychloroquine (HCQ), lopinavir-ritonavir combination therapy (LPV/r), ICU care, and non-invasive ventilation (NIV) was also collected. Outcome of COVID-19-infected patients was measured by the number of recovered patients, duration of seroconversion (duration from first positive to first negative RT-PCR for COVID-19), duration of hospital stays, and number of deaths during the course of treatment. The collected data was compiled, tabulated, interpreted, and correlated in both the groups to establish differences in COVID19 manifestations on the basis of glycemic control in T2DM.

\section{Statistical analysis}

The descriptive statistics for quantitative data was expressed as mean and standard deviation, and qualitative data was 
expressed as proportions. The parameters were compared among different groups using chi-square and z-score test for significant differences. The level of significance was assigned at a $p$ value less than 0.05 .

\section{Results}

In the present study, 22 patients $(27.50 \%)$ had poorly controlled blood sugar levels with HbAlc level more than $8 \mathrm{~g} \%$ and belonged to group 1, whereas 58 patients $(72.50 \%)$ had controlled blood sugar levels with HbA1c level less than $8 \mathrm{~g} \%$ that belonged to group 2. The mean age of SARS-CoV-2infected patients with T2DM was 61.45 years $(95 \% \mathrm{CI}$ : $61.45 \pm 5.08, \mathrm{SD}=11.59)$. Mean age of uncontrolled diabetes group was 63.2 years $(95 \% \mathrm{CI}: 63.2 \pm 4.35, \mathrm{SD}=4.96)$, whereas mean age in controlled diabetes group was 60.86 years (95\% CI: $60.86 \pm 6.67, \mathrm{SD}=13.18$ ), and the difference was non-significant $(p=0.489)$ (Table 1). There were no significant gender differences among the groups $(p=$ 0.301). Other comorbidities with COVID-19 have not been considered to understand entirely the impact of uncontrolled glycemic condition on predisposition and severity in COVID19-infected patients.

Clinical presentation Overall, 24 patients (30.00\%) had symptomatic presentation. Group $1(50.00 \%)$ exhibited a significantly greater $(p=0.0164)$ symptomatic presentation as compared with group 2 (22.41\%). Most of the COVID-19-infected patients with T2DM presented with cough (16.25\%), fever $(15.00 \%)$, shortness of breath $(11.25 \%)$, and sore throat $(8.75 \%)$. A few patients also reported for headache $(5.00 \%)$, chest pain (3.75\%), and symptoms $(5.00 \%)$ like pain in the abdomen, vomiting, diarrhea, and altered sensorium. Cough (31.82\% in group 1 vs $10.34 \%$ in group 2) and shortness of breath $(22.73 \%$ in group 1 vs $6.90 \%$ in group 2$)$ were found more often $(p<0.05)$ in uncontrolled diabetes group compared with controlled diabetes group with $p$ values 0.0203 and 0.0455 , respectively.

Laboratory findings (Table 1) The blood profile of both the groups showed normal total leukocyte counts (TLC) with an average TLC $7.48 \times 10^{9} / \mathrm{L} \pm 4.23$, platelet count $2.12 \mathrm{Lac} / \mu \mathrm{l}$ \pm 0.64 , and neutrophil/lymphocyte $(\mathrm{N} / \mathrm{L})$ ratio $3.15 \pm 1.98$. However, uncontrolled diabetes group showed higher TLC and N/L ratio, i.e., $9.01 \times 10^{9}$ and 3.98 , as compared with controlled group with values $7.01 \times 10^{9}(p=0.0403)$ and $3.00(p=0.0495)$. The other laboratory parameters taken into consideration were as follows: c-reactive protein, ferritin, fibrin degradation product, D-dimer, and interleukin-6. The biochemical parameters were found to be more significantly variable among the two groups of patients. The parameters such as FDP $43.22 \mu \mathrm{g} / \mathrm{L}$ vs $24.69 \mu \mathrm{g} / \mathrm{L}(p=0.0433)$, D-dimer
$6.78 \mu \mathrm{g} / \mathrm{L}$ vs $4.05 \mu \mathrm{g} / \mathrm{L}(p=0.047)$, and IL-6 levels $78.52 \mathrm{pg} / \mathrm{mL}$ vs $50.32 \mathrm{pg} / \mathrm{mL}(p=0.0256)$ were observed to be elevated in group I patients in comparison with group II patients.

Radiological findings For the assessment of lung involvement in sample population, digital chest radiograph (CXR) of all patients, USG chest of 16 patients $(20.00 \%)$, and HRCT chest of 30 patients $(37.50 \%)$ were available. CXR represented classic for COVID-19 images in 20 patients $(25.00 \%)$ with an average visual score of $0.78 \pm 0.90$ out of 4 . USG severity score was $16.14 \pm 11.32$ out of 42 . The average CT severity score was $7.4 \pm 6.75$ out of 25 with CT severity score $>10 / 25$ in 12 patients $(40.00 \%)$. CXR average visual score was significantly $(p=0.0439)$ higher in group $1(1.12)$ than group 2 (0.68). CT severity score was significantly $(p=0.0489)$ higher in uncontrolled diabetes group (11.96) as compared with controlled diabetes group (6.1) with a CT severity score $>10 / 25$ in $75.00 \%$ and $27.27 \%$ patients in respective groups $(p=$ 0.0182).

Diabetic complications Six patients (7.50\%) developed diabetic ketoacidosis (DKA), and nine patients $(11.25 \%)$ had septic shock. DKA developed more often $(p=0.0257)$ in patients of uncontrolled diabetes (18.18\%) as compared with the controlled diabetes group (3.45\%). Septic shock was observed in $22.73 \%$ patients of uncontrolled diabetes, whereas it was $6.90 \%$ in controlled diabetes group.

Analysis of severity, management, and treatment in the two groups An observed comparison of uncontrolled and controlled glycemic patients showed that uncontrolled diabetic patients $(68.18 \%)$ required insulin therapy more often $(p=0.0226)$ than the controlled group $(39.66 \%)$. Requirement of non-invasive ventilation was significantly more in uncontrolled diabetes group as compared to controlled diabetes group patients $(p=0.0292)$, along with higher ICU care requisite for group I, whereas the need of LPV/r combination therapy for COVID-19 treatment in standard dosage did not differ significantly $(p=0.1498)$ between the two groups.

Outcome In total, 80 patients were considered; out of that, 40 patients $(50.00 \%)$ recovered, whereas 14 patients $(17.50 \%)$ succumbed to COVID-19, and remaining patients were undergoing treatment until the date this study was compiled. Average duration of hospital stays was significantly prolonged ( $p=0.0479$ ) in patients with uncontrolled diabetes (16.94 days) as compared with patients with controlled diabetes (13.91 days). As observed from the collected data, the average recovered patients of uncontrolled diabetic group were very low $(36.36 \%)$ in comparison with group 2 $(55.17 \%)$. Not only this, significantly, higher COVID-19- 


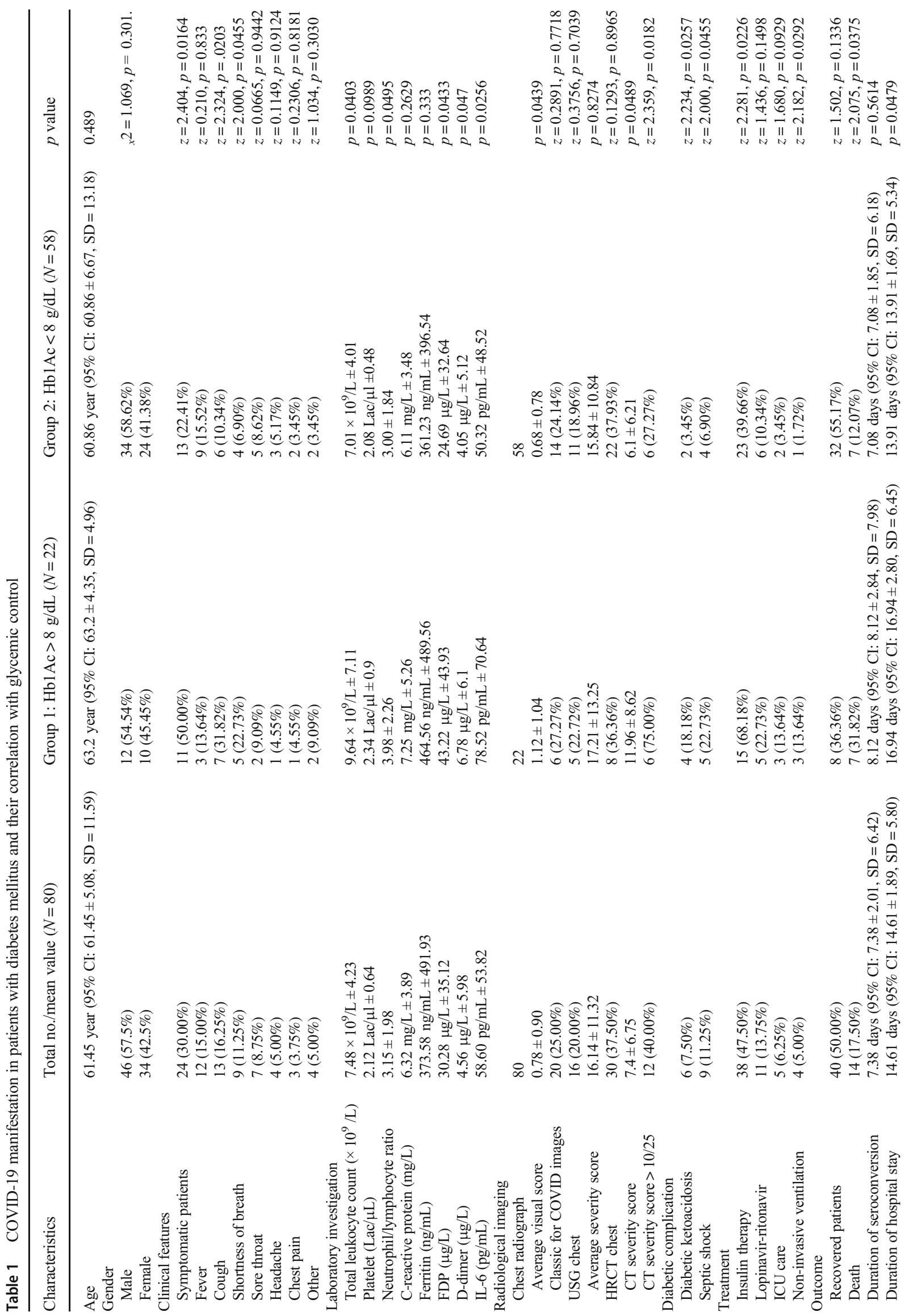


related mortality was observed in uncontrolled diabetes group (31.82\% vs $12.07 \%)$.

\section{Discussion}

In our study, we have observed that uncontrolled diabetes has significantly altered the biochemical parameters along with worsening of prognosis. Diabetes mellitus predisposes an individual to a certain type of infection and mortality [12] including the COVID-19, although its risk as associated comorbidity in COVID-19 needs further exploration. The prevalence of T2DM in India is $7.3 \%$ [13], thereby predisposing a large proportion of population to COVID-19 and its complications. Type 2 diabetes mellitus as a consequence of metabolic syndrome and obesity predisposes to immune dysfunction with raised inflammatory factors and chemokines [14, 15]. ACE2 possessing anti-inflammatory property has been linked to SARS-CoV-2 invasion in human cells, and its expression is found reduced in patients of diabetes mellitus, possibly due to glycosylation [16]. This might explain higher predisposition of COVID-19 patients with diabetes mellitus to severe acute lung injury and ARDS [16]. Immunostaining technique has revealed an enhanced staining characteristic for ACE2 in islet tissue as compared with the exocrine pancreatic tissues suggesting a plausible role of coronavirus in islet destruction [17]. Thus, COVID-19 might lead to a sharp fluctuation in blood glucose level in diabetes patients, adversely affecting the varied clinical presentation of the disease.

In the present study, clinical presentation was more pronounced in uncontrolled diabetes group as compared with controlled diabetes group. Cough and shortness of breath occurred more often in uncontrolled diabetes patients, and the underlying cause for such presentation might be early and extensive lung involvement in COVID-19 infection due to glycemic variation. This was evident by baseline CXR findings of the study that suggested a higher proportional lung involvement in uncontrolled diabetes group as compared with controlled diabetes group. Moreover, a high CT severity score also indicated extensive lung involvement in uncontrolled diabetic patients. The aforementioned clinical picture supported by radiological findings suggested a severe pneumonia in uncontrolled diabetic patients as compared with the controlled diabetic patients.

The average total leukocyte count was observed higher in uncontrolled diabetes that could be attributable to an increased secondary inflammatory response in these patients. Neutrophil to lymphocyte ratio was also raised in peripheral blood of uncontrolled diabetes patients, possibly due to neutrophilia or a relative lymphocytopenia as a consequence of COVID-19 infection. Furthermore, the serum levels of inflammatory biomarkers such as IL-6, ferritin, and CRP were alarmingly raised in uncontrolled diabetes group. IL-6 is a predictor of disease severity and prognosis [18], and in a study conducted by Huang et al., it was reported that levels of IL-6 were elevated along with significantly reduced lymphocyte count, in patients with SARS-CoV-2 infection [19]. Excessively raised ferritin level is an indicator of activation of the monocyte-macrophage system that contributes significantly to the inflammatory storm associated with COVID-19 [20]. In the present study, raised ferritin levels were observed in uncontrolled diabetes, suggesting a higher susceptibility of such patients for an inflammatory storm, responsible for rapid deterioration due to COVID-19. Inflammation-associated hypoxia might induce thrombin activation with a consequent unfolding of exogenous coagulation pathway [20]. Inflammatory storm in COVID-19 is associated with significant rise in D-dimer levels, and similar pattern has been observed in our study as well where uncontrolled glycemic patients had significantly increased levels of D-dimer as compared with controlled diabetic patients. This finding is an indication of a hypercoagulable state and even disseminated intravascular coagulation in such patients. Patients of uncontrolled diabetes mellitus were more prone to develop diabetic complication during the natural course of COVID-19 as compared with the controlled diabetes group. Patients with uncontrolled diabetes developed diabetic complication like diabetic ketoacidosis and septic shock, more often.

Patients with uncontrolled diabetes mellitus required aggressive pharmacological and supportive treatments compared with controlled diabetes group as is much evident from more frequent requirement of insulin therapy in group I. Moreover, need of supportive therapies such as non-invasive ventilation and ICU care was also higher in uncontrolled diabetes patients. A low recovery rate, high mortality, and prolonged hospitalization indicated poor outcome in patients with uncontrolled diabetes mellitus as compared with control glycemic group of diabetes mellitus.

\section{Conclusion}

In the current retrospective study, we have considered 80 COVID-19-infected patients with T2DM and characterized them with biochemical, radiological, and other required clinical parameters. From the various observations, it can be concluded that uncontrolled diabetes mellitus may predispose an individual to a severe and fatal COVID-19 infection. The severity of COVID-19 in diabetics could be attributable to the dysfunctional immune system, with a simultaneous susceptibility to viral infection and an exaggerated immune response like cytokine storm. Such a status of immunity provides a favorable condition for viral survival and longer recovery duration in diabetics. COVID-19 patients with uncontrolled diabetes require higher attention in terms of pharmacological and supportive treatment than those with controlled diabetes 
group. A high prevalence of T2DM in India predisposes a large proportion of population to COVID-19 and its complications. HbA1c more than $8 \mathrm{~g} \%$ in patients with uncontrolled diabetes mellitus should be considered a risk factor for an overall higher susceptibility for COVID-19 infection and severity in terms of symptomatic presentation, inflammatory storm, rapid pulmonary invasion, requirement of more intensive treatment, and a poor outcome. Hence, an extreme care for preventing COVID-19 in people with underlying DM is advisable beside an intensive care in already infected individuals.

Acknowledgments We would like to acknowledge Dr. Abhishek Agrawal, Dr. C. L. Nawal, Dr. S. Banerjee, Dr. Prakash Keswani, Dr. Sunil Mahavar, Dr. R S Chejara, Dr. Vidyadhar Singh, Dr. Kapil, Dr. Amitabh Dube, Dr. Bhupendra Patel, Dr. Meenu Bagarhatta, and team of the Department of General Medicine and Department of Radiodiagnosis, SMS Medical College and Attached Group of Hospitals, Jaipur, Rajasthan, India, for their valuable support and for providing radiological information of COVID-19 patients.

Authors' contributions S. Bhandari, G. Rankawat, and A. Singh formulated the research questions, designed the study, developed the preliminary search strategy, and drafted the manuscript; G. Rankawat and A. Singh collected and analyzed the data for study. G. Rankawat wrote the manuscript. S. Kakkar and V. Gupta conducted the quality assessment. All authors critically reviewed the manuscript for relevant intellectual content. All authors have read and approved the final version of the manuscript.

Data availability Available from the corresponding author upon reasonable request.

\section{Compliance with ethical standards}

Conflict of interest The authors declare that they have no conflict of interest.

Ethical approval This study was approved by the ethical and research committee of the SMS Medical College Hospital, Jaipur, India. All ICMR guidelines have been duly followed.

\section{References}

1. Chen N, Zhou M, Dong X, Qu J, Gong F, Han Y, et al. Epidemiological and clinical characteristics of 99 cases of 2019 novel coronavirus pneumonia in Wuhan, China: a descriptive study. Lancet. 2020;395(10223):507-13.

2. Hui DS, Azhar E, Madani TA, Ntoumi F, et al. The continuing 2019-nCoV epidemic threat of novel coronaviruses to global health - The latest 2019 novel coronavirus outbreak in Wuhan, China. Int J Infect Dis. 2019;91:264-6.

3. Murthy S, Gomersall CD, Fowler RA. Care for critically ill patients with COVID19. JAMA. 2020;323(15):1499.
4. WHO Coronavirus Disease (COVID-19) Dashboard. https:// covid19.who.int/

5. Kuba K, Imai Y, Rao S, Gao H, Guo F, Guan B, et al. A crucial role of angiotensin converting enzyme 2 (ACE2) in SARS coronavirusinduced lung injury. Nat Med. 2005;11(8):875-9.

6. Wrapp D, Wang N, Corbett KS, Goldsmith JA, Hsieh CL, Abiona $\mathrm{O}$, et al. Cryo-EM structure of the 2019-nCoV spike in the prefusion conformation. Science. 2020;367:1260-3.

7. Knapp S. Diabetes and infection: is there a link mini-review. Gerontology. 2013;59(2):99-104.

8. Hodgson K, Morris J, Bridson T, Govan B, Rush C, Ketheesan N. Immunological mechanisms contributing to the double burden of diabetes and intracellular bacterial infections. Immunology. 2015;144(2):171-85.

9. Warren MA, Zhao Z, Koyama T, Bastarache JA, Shaver CM, Semler MW, et al. Severity scoring of lung edema on the chest radiograph is associated with clinical outcomes in ARDS. Thorax. 2018 Sep;73(9):840-6.

10. Peng QY, Wang XT, Zhang LN. Chinese Critical Care Ultrasound Study Group (CCUSG). Findings of lung ultrasonography of novel corona virus pneumonia during the 2019-2020 Epidemic. Intensive Care Med. 2020;46(5):849-50.

11. Rodriguez-Morales AJ, Cardona-Ospina JA, Gutiérrez-Ocampo E, Villamizar-Peña R, et al. Clinical, laboratory and imaging features of COVID-19: a systematic review and meta-analysis. Travel Med Infect Dis. 2020 Mar;13:101623.

12. Yang JK, Feng Y, Yuan MY, Yuan SY, Fu HJ, Wu BY, et al. Plasma glucose levels and diabetes are independent predictors for mortality and morbidity in patients with SARS. Diabet Med. 2006 Jun;23(6):623-8.

13. Shu CJ, Benoist C, Mathis D. The immune system's involvement in obesity-driven type 2 diabetes. Semin Immunol. 2012;24(6):43642.

14. Anjana RM, Deepa M, Pradeepa R, Mahanta J, Narain K, Das HK, et al. Prevalence of diabetes and prediabetes in 15 states of India: results from the ICMR-INDIAB population-based cross-sectional study. Lancet Diab Endocrinol. 2017;5:585-96.

15. Meshkani R, Vakili S. Tissue resident macrophages: key players in the pathogenesis of type 2 diabetes and its complications. Clin Chim Acta. 2016;462:77-89.

16. Yang JK, Lin SS, Ji XJ, Guo LM. Binding of SARS coronavirus to its receptor damages islets and causes acute diabetes. Acta Diabetol. 2010;47(3):193-9.

17. Tikellis $\mathrm{C}$, Thomas MC. Angiotensin-converting enzyme 2 (ACE2) is a key modulator of the renin angiotensin system in health and disease. Int J Pept. 2012;2012:1-8.

18. Tanaka T, Narazaki M, Kishimoto T. Immunotherapeutic implications of IL- 6 blockade for cytokine storm. Immunotherapy. 2016;8(8):959-70.

19. Huang C, Wang Y, Li X, Ren L, Zhao J, Hu Y, et al. Clinical features of patients infected with 2019 novel coronavirus in Wuhan. China Lancet. 2020;395(10223):497-506.

20. Guo W, Li M, Dong Y, et al. Diabetes is a risk factor for the progression and prognosis of COVID-19. Diabetes Metab Res Rev. 2020;e3319. https://doi.org/10.1002/dmrr.3319.

Publisher's note Springer Nature remains neutral with regard to jurisdictional claims in published maps and institutional affiliations. 